outcomes for small and medium aneurysms for aneurysm locations including internal carotid artery (ICA) and distal to ICA terminus are unknown.

Methods The EVOLVE study is a prospective, historical-control based, single arm, multicenter clinical trial delineating the safety and efficacy of the second generation Surpass EVOLVE Flow Diverter System for the treatment of wide-neck $(\geq 4 \mathrm{~mm}$ or no discernible neck), small and medium sized saccular or fusiform IA $\leq 12 \mathrm{~mm}$ located on the ICA and its branches with a reference vessel diameters 2.5 to $5.0 \mathrm{~mm}$.

Approximately 235 patients will be enrolled at approximately 40 investigational sites located in the USA, Canada, and Australia. Clinical follow-up is planned at discharge, 30 and 180 days and at 1, 2, and 3 years. All subjects will undergo DSA imaging at 1 - and 3 -years post device implantation. The respective primary safety and efficacy endpoints are neurological death or disabling stroke at 12 months, as assessed by an independent CEC; and the composite of Raymond I occlusion with no significant (>50\%) parent artery stenosis, and no target aneurysm retreatment; as assessed by an independent Core Lab. An analysis of non-inferiority to pre-established safety and effectiveness performance goals will be completed following 12-month follow-up.

Other key outcomes include assessment of procedural technical success, major stroke, minor stroke, TIA, stent thrombosis, recanalization, durability of occlusion and occlusion status. Conclusion The EVOLVE prospective, multicenter, single arm, pre-market study (ClinicalTrials.gov NCT: NCT04195568), is designed to evaluate the safety, efficacy, and performance of the Surpass EVOLVE Flow Diverter System in the treatment of unruptured wide-neck, saccular or fusiform IA located in the ICA and its branches with an RVD of 2.5 to $5.0 \mathrm{~mm}$.

Disclosures A. Arthur: 2; C; Stryker Neurovascular. J. Fifi: 1; C; Stryker Neurovascular. 2; C; Stryker Neurovascular. H. Rice: 2; C; Stryker Neurovascular. A. Wakhloo: 2; C; Stryker Neurovascular. A. Coon: 2; C; Stryker Neurovascular. V. Pereira: 2; C; Stryker, Medtronic, Balt, Cerenovus.

\section{E-035 SAFETY AND EFFICACY OF STENT-ASSISTED COILING OF RUPTURED WIDE NECK ANEURYSMS IN COMPARISON TO BALLOON ASSISTED COILING IN ACUTE SUBARACHNOID HEMORRHAGE} ${ }^{3} \mathrm{~A}$ Mendez Ruiz, ${ }^{3} \mathrm{C}$ Zevallos, ${ }^{3} \mathrm{D}$ Quispe-Orozco, ${ }^{3} \mathrm{M}$ Farooqui, ${ }^{2} \mathrm{~J}$ Delgado Almandoz, ${ }^{3} \mathrm{~S}$ Ortega-Gutierrez*. ${ }^{1}$ Aurora Neuroscience Institute, Milwaukee, WI; ${ }^{2}$ Abbott Northwestern Hospital, Minneapolis, MN; ${ }^{3}$ University of lowa Hospitals and Clinics, lowa City, IA; ${ }^{4}$ University of Pittsburgh Medical Center, Pittsburgh, PA

10.1136/neurintsurg-2021-SNIS.131

Introduction Ruptured wide neck aneurysms (RWA) have proven challenging for acute endovascular embolization and are still preferentially treated with microsurgical clipping in most institutions. Balloon assisted coiling (BAC) has been used for acute embolization of RWA with some success in recent past but with high recanalization rates requiring re-treatment, risk of parent vessel coil protrusion, and thromboembolic complications. The aim of this study is to assess the safety and efficacy of using Stent assisted coiling (SAC) and/or Balloon assisted coiling (BAC) for acute embolization of RWA.

Methods We performed a retrospective analysis of data at two comprehensive stroke centers (CSC) for patients with RWA who underwent endovascular treatment with SAC and BAC between 2010 and 2020. Patient selection for either treatment category was at the discretion of the treating physician. The treating physician also made the decision in case of SAC, whether to load the patient with dual oral antiplatelets or place them on intravenous antiplatelet regimen. Primary clinical outcomes of interest were modified Rankin Scale (mRS) at 3-months and Raymond Roy (RR) Occlusion Classification, immediately post-operative and at 3 -months. Patient demographics, clinical presentation (Hunt and Hess score $\{\mathrm{HH}$, modified Fisher scale $\{\mathrm{mFS}\}$ and Glasgow coma scale $\{\mathrm{GCS}\}$ ), comorbidities, aneurysms characteristics, technical success, complications, and clinical and imaging follow-ups were also assessed. Univariate and multivariable logistic regression and ordinal regression was used to compare the primary and secondary outcomes. All the statistical analysis was performed using R-software at an $\alpha \leq 0.05$ significance.

Results 112 patients were treated with SAC and 109 underwent BAC. There was no significant difference in baseline characteristics between the 2 groups with median age of 53.5 years in SAC and 55.81 years in BAC $(p=0.419)$, nor in their clinical characteristics with regards to $\mathrm{HH}, \mathrm{mFS}$, and GCS. There was no significant difference between the two groups with regards to aneurysm location $(77.7 \%$ vs $84.4 \%$ in anterior circulation, $\mathrm{p}=0.78$ ) or size. Coil herniation was significantly higher in BAC group $(9.2 \%$ vs $1.8 \%, \mathrm{p}=0.033)$ with no significant difference in other perioperative and in-hospital complications between the two groups including intracranial hemorrhages, strokes, groin hematoma requiring transfusions, and EVD related complications. There was no significant difference in mRS 3-6 at 3 months (38 vs 22, p=0.096). The SAC group had significantly higher number of patients with RR Class I, immediately post-operative (45 vs 26, $\mathrm{p}<0.001 \%$ ), with significantly less patients in RR Class II and III at 3 months follow up (29 vs $39, \mathrm{p}<0.001 \%)$. Similarly, after adjusting for the variables, there was no statistical difference in the mRS shift analysis $(p=0.062)$ and $R R(p=0.35)$ at 90 days among the two groups.

Conclusion In this study, we observed that SAC is as safe as BAC for acute treatment of RWA, with better aneurysm occlusion rates, less re-treatment and without an increase in hemorrhagic complications. Further studies are warranted to validate these findings in a prospective randomized manner before widespread application in the management of patients with acute aneurysmal subarachnoid hemorrhage.

Disclosures S. Dandapat: None. A. Wallace: None. G. Lopez: None. Y. Kayan: None. A. Mendez Ruiz: None. A. Copelan: None. A. Dajles: None. A. Mendez Ruiz: None. C. Zevallos: None. D. Quispe-Orozco: None. M. Farooqui: None. J. Delgado Almandoz: None. S. Ortega-Gutierrez: None.

\section{E-036 NEUROENDOVASCULAR INTERVENTIONS WITH TRANSCIRCULATION APPROACH: A CASE-SERIES APPROACH TO SOLVE CHALLENGING SITUATIONS}

${ }^{1} \mathrm{M}$ Colasurdo*, ${ }^{2} \mathrm{G}$ Edhayan, ${ }^{3} \mathrm{P}$ Kan, ${ }^{1} \mathrm{~K}$ Raghuram. ${ }^{1}$ Neuroradiology, University of Texas Medical Branch, Galveston, TX; ${ }^{2}$ Radiology, University of Texas Medical Branch, Galveston, TX; ${ }^{3}$ Neurosurgery, University of Texas Medical Branch, Galveston, TX

10.1136/neurintsurg-2021-SNIS. 132

Introduction With the development of advanced endovascular techniques and materials, neurointerventionalists are able to perform challenging and complex cases that were previously difficult to perform. Transcirculation approaches could be a 


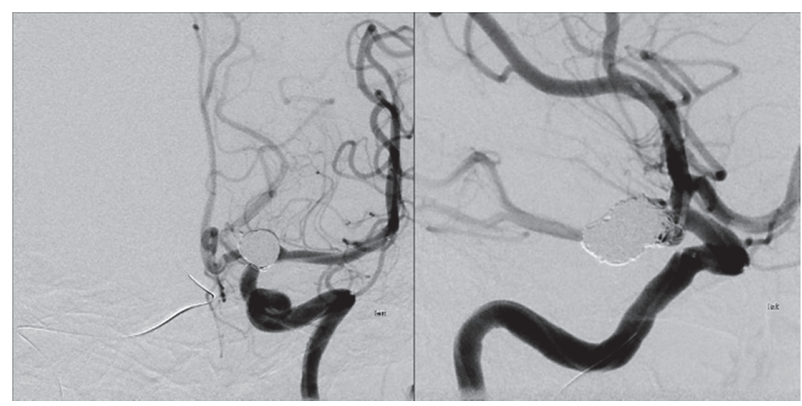

Abstract E-036 Figure 1

tool used in these cases, providing access to the target vessel through the contralateral or opposite circulation when anterograde access is difficult or non-achievable.

Materials and methods Cases of transcirculation intracranial interventions performed between two institutions (Tampa General Hospital, Tampa, FL and University of Texas Medical Branch, Galveston, TX) were reviewed, which included four cases of aneurysm and arteriovenous malformation (AVM) embolization. Complications, angiographic results, and clinical outcomes were assessed.

Results Successful transcirculation neurointerventions were performed in four cases. These cases include a posterior communicating (Pcomm) artery aneurysm which was coiled with M1 to Pcomm stent assistance, a basilar tip aneurysm which was coiled with P1 to contralateral P1 stent assistance, a complex posterior fossa AVM, and a giant left cavernous internal carotid artery (ICA) aneurysm. The posterior fossa AVM was embolized through a recurrent superior cerebellar artery retrogradely catheterized via the contralateral posterior communicating artery. The left cavernous ICA aneurysm was initially unsuccessfully treated with an anterograde approach and was retreated with a combined anterograde and retrograde approach through the contralateral anterior circulation across the anterior communicating artery with subsequent successful positioning of multiple flow diverters. In three cases the anterior or posterior circulation were accessed through the posterior communicating arteries, and in one case the contralateral anterior circulation was accessed by crossing the anterior communicating artery.

Conclusions Transcirculation approaches can be performed for cases where conventional treatment options are not feasible or as a bailout strategy in failed treatment attempts. In this small case series, it seemed a safe and efficient way of facing challenging cases.

Disclosures M. Colasurdo: None. G. Edhayan: None. P. Kan: None. K. Raghuram: None.

\section{E-037 ANGIOGRAPHIC INVESTIGATION OF ORBITAL VASCULAR VARIATIONS IN THE RABBIT: IMPLICATIONS FOR INTRA- ARTERIAL CHEMOTHERAPY MODELS}

${ }^{1} \mathrm{M}$ Feldman*, ${ }^{2} \mathrm{~B}$ Poitras, ${ }^{3} \mathrm{~A}$ Daniels, ${ }^{1} \mathrm{M}$ Froehler. ${ }^{1}$ Neurosurgery, Vanderbilt University Medical Center, Nashville, $T_{N}{ }^{2}$ Radiology, Vanderbilt University, Nashville, $T N_{;}{ }^{3}$ Department of Ophthalmology and Visual Sciences, Vanderbilt University Medical Center, Nashville, TN

\subsection{6/neurintsurg-2021-SNIS.133}

Introduction/Purpose The New Zealand White rabbit (NZWR) is the first small-animal experimental model of intra-arterial chemotherapy (IAC) for retinoblastoma treatment. The NZWR has dual ophthalmic arteries (OA): the external OA (EOA) arises from the external carotid artery and the internal OA (IOA) from the internal carotid artery. We describe the technique that we have refined for $\mathrm{OA}$ catheterization in rabbits, and describe the angioanatomical variations in the $\mathrm{OA}$ supply to the NZWR eye and implications for IAC delivery, which were identified as part of a project exploring IAC effects in a rabbit retinoblastoma model.

Materials and Methods We developed a novel technique to perform angiography of the external and internal carotid arteries and superselective angiography of the EOA and IOA in NZWR using transfemoral access and a microwire/microcatheter system that closely resembled our human IAC paradigm. EOA and IOA supply to the eye was determined angiographically and recorded before selective OA catheterization and angiography.

Results 114 rabbits underwent carotid angiographic evaluation and OA catheterization (161 total eyes evaluated, 112 right, 49 left). Most eyes had a single dominant arterial supply; either IOA or EOA. EOA was dominant in 73\% (118/161), and IOA was dominant in $17 \%(27 / 161)$. Co-dominant supply was seen in $10 \%(16 / 161)$. Of the rabbits with bilateral OA catheterization, 25/47 (53\%) had bilateral dominant EOA.

Conclusions Successful catheterization of the $\mathrm{OA}$ in the NZWR can be readily accomplished with nuanced technique that mimics that used in humans. The external $O A$ is the dominant arterial supply in the majority of NZWR eyes. These findings allow for successful reproduction of OA catheterization studies of IAC for retinoblastoma in NZWR. These findings may also give insight into ICA and ECA-targeting IAC techniques in humans.

Disclosures M. Feldman: None. B. Poitras: 5; C; Cerenovus. A. Daniels: None. M. Froehler: 1; C; Genentech, Medtronic, Stryker, Microvention, and Penumbra. 2; C; Genentech, Medtronic, Stryker, Balt USA, Viz.ai, and Corindus.

\section{E-038 THROMBECTOMY OUTCOMES AND OBSERVATIONS ON DENTAL HYGIENE (TOOTH)}

${ }^{1} \mathrm{M}$ Feldman*, ${ }^{2} \mathrm{~S}$ Koester, ${ }^{3} \mathrm{R}$ Chaliff, ${ }^{1} \mathrm{M}$ Longo, ${ }^{1} \mathrm{~A}$ Yengo-Kahn, ${ }^{4} \mathrm{M}$ Froehler, ${ }^{1} \mathrm{R}$ Chitale. ${ }^{1}$ Neurosurgery, Vanderbilt University Medical Center, Nashville, $T N_{i}{ }^{2}$ Vanderbilt University School of Medicine, Nashville, TN; ${ }^{3}$ Oral and Maxillofacial Surgery, Vanderbilt University Medical Center, Nashville, TN; ${ }^{4}$ Cerebrovascular Diseases Program, Vanderbilt University Medical Center, Nashville, TN

\subsection{6/neurintsurg-2021-SNIS.134}

Introduction Previous literature has identified an association between poor dentition and risk for stroke. This association has not been identified for acute stroke outcomes after endovascular treatment. We aimed to assess whether tooth loss and dental status was predictive of poor outcomes after thrombectomy for acute ischemic stroke.

Methods A retrospective review was conducted on all adult patients at a single comprehensive stroke center who underwent thrombectomy between 2012 to 2018. Inclusion criteria included complete CT imaging where dentition could be assessed for the front 28 teeth. Demographic, comorbidity, and outcome data were also collected for all of these patients. A multivariate analysis was performed, with the primary outcome as post-operative MRS.

Results 273 consecutive patients were included in our analysis. The average age was $62.96(+/-15.76)$ and $137(50 \%)$ of the patients were male. Patients missing all teeth did not 